\title{
Minimal energy packings of nearly flexible polymers
}

\author{
Robert S. Hoy ${ }^{1}$ ? Jared Harwayne-Gidansky ${ }^{2}$, and Corey S. O'Hern ${ }^{3,4,5,6}$ \\ ${ }^{1}$ Department of Physics, University of South Florida, Tampa, FL 33620 and \\ Departments of Electrical Engineering ${ }^{2}$, Mechanical Engineering \& Materials Science $^{3}$, Applied Physics ${ }^{4}$, Physics ${ }^{5}$, \\ and ${ }^{6}$ Integrated Graduate Program in Physical and Engineering Biology, Yale University, New Haven, CT 06520
}

(Dated: June 24, 2018)

\begin{abstract}
We extend recent studies of the minimal energy packings of short flexible polymers with hardcore-like repulsions and short-range attractions to include bond-angle interactions with the aim of describing the collapsed conformations of 'colloidal' polymers. We find that flexible tangent stickyhard-sphere (t-SHS) packings provide a useful perturbative basis for analyzing polymer packings with nonzero bending stiffness only for small ratios of the stiffnesses for the bond-angle $\left(k_{b}\right)$ and pair $\left(k_{c}\right)$ interactions, i.e. $k_{b}^{\text {crit }} / k_{c} \lesssim 0.01$ for $N<10$ monomers, and the critical ratio decreases with $N$. Below $k_{b}^{\text {crit }}$, angular interactions give rise to an exponential (in $N$ ) increase in the number of distinct angular energies arising from the diversity of covalent backbone paths through t-SHS packings. As $k_{b}$ increases above $k_{b}^{\text {crit }}$, the low-lying energy landscape changes dramatically as finite bending stiffness alters the structure of the polymer packings. This study lays the groundwork for exact-enumeration studies of the collapsed states of t-SHS-like models with larger bending stiffness.
\end{abstract}

PACS numbers: $64.70 . \mathrm{km}, 81.16 . \mathrm{Dn}, 02.10 . \mathrm{Ox}, 87.15 . \mathrm{hp}$

\section{INTRODUCTION}

Protein folding and other examples of polymer collapse in dilute solutions are complex processes that involve the cooperative motion of thousands of atoms. A number of early studies of polymer and protein structure and dynamics employed hard-sphere models with only steric interactions, bond-length, and bond-angle constraints to understand polymer elasticity [1] as well as secondary structure 2] and packing in hydrophobic cavities [3] in proteins. More recent studies have implemented Monte Carlo, Brownian dynamics, and molecular dynamics (MD) simulations that include excluded volume and stereochemical constraints as well as solventmediated attractive interactions to investigate numerous examples of polymer collapse. However, recent advances in colloidal synthesis and self assembly have enabled studies of "colloidal polymers" with hard-core-like repulsions, short-range attractions, and much greater chain flexibility than typical synthetic or biological polymers [4, 5], which has prompted renewed interest in simple polymer models. [6 15] We seek to develop the ability to theoretically predict which structures are most likely to form for a given set of interactions and solvent conditions, and thus reducing the need for trial-and-error synthesis of self-assembled nanostructures.

The ground state packings of short, flexible tangent sticky hard sphere (t-SHS) polymers have recently been characterized via complete enumeration. 16] These packings possess a wider range of symmetries and shapes than the ground state packings of polymers with longer-ranged attractive (e.g. Lennard-Jones [17]) pair potentials. Further, the number $N_{\text {micro }}$ of distinguishable, energetically

*Electronic address: rshoy@usf.edu degenerate ground-state flexible t-SHS packings ("microstates") grows exponentially with the polymerization index $N$, with a corresponding increase in the diversity of paths followed by the covalent backbones.

In this manuscript, we characterize the ground state packings for short, semi-flexible t-SHS polymers with finite bending stiffness $k_{b}$. In particular, we calculate the potential energy of semi-flexible polymer packings as a function of $k_{b}$ and identify the maximum bending stiffness $k_{b}^{\text {crit }}$ below which the reference set of ground-state flexible t-SHS packings are the lowest energy structures. We also examine the structural differences between the lowest energy semi-flexible structures and the completely flexible reference set that appear as $k_{b}$ approaches and exceeds $k_{b}^{c r i t}$. The crossover occurs in a range of $k_{b}$ lying between estimates of $k_{b}^{\text {crit }}$ from a simple analytic criterion and results from MD simulations of polymer collapse. In this range of $k_{b}$, the low-lying energy minima in the landscape change dramatically. In general, states with $k_{b}>k_{b}^{\text {crit }}$ are less collapsed than flexible tSHS packings since it is energetically unfavorable for such chains to form the maximum number of pair contacts allowed by steric constraints. Our results will inform future exact-enumeration studies of polymer packings with larger bending stiffness.

\section{MODEL AND METHODS}

We consider a polymer model with harmonic pair and bond-angle potentials with respective "spring constants" $k_{c}$ and $k_{b}$, and equilibrium bond angle $\theta_{e q}$ (Fig. 2 and Eqs. 2.3). The ground-state flexible $\left(k_{b}=0\right)$ t-SHS polymer packings can provide a useful reference set of configurations for studying the lowest energy semi-flexible $\left(k_{b}>0\right)$ t-SHS polymer packings when there is a oneto-one mapping from the $N_{\text {micro }}$ ground state polymer 


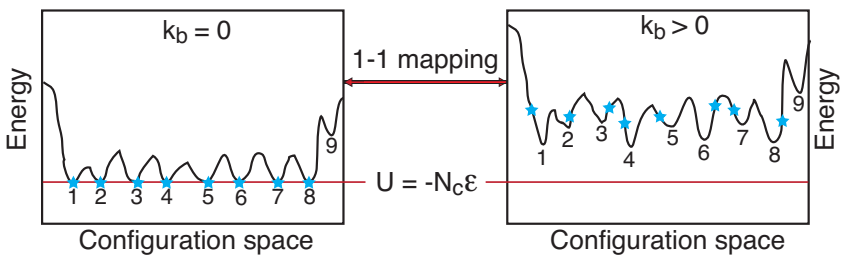

FIG. 1: Schematic of the low-lying minima in the energy landscape for polymer packings as a function of the configurational degrees of freedom $\{\vec{r}\}$. For vanishing bending stiffness $k_{b}=0$ (left), the ground-state flexible polymer packings (stars) are energetically degenerate and correspond to the lowest $N_{\text {micro }}$ inherent structures (minima of the black curve). Increasing $k_{b}$ (right) deforms the energy landscape and raises the energies of the $N_{\text {micro }}$ minima that correspond to the flexible polymer packings. For $k_{b}<k_{b}^{\text {crit }}$, the $N_{\text {micro lowest energy semi- }}$ flexible polymer packings correspond to the $N_{\text {micro }}$ groundstate flexible polymer packings.

packings for $k_{b}=0$ to the $N_{\text {micro }}$ lowest energy polymer packings with finite bending stiffness. In this case, the $N_{\text {micro }}$ zero- $k_{b}$ t-SHS packings lie within the basins [18] of the $N_{\text {micro }}$ lowest-energy packings at finite $k_{b}$ as shown in Fig. 1 (right). Increasing $k_{b}>0$ deforms the energy landscape, raises the energies of the $N_{\text {micro }}$ flexible polymer packings, and breaks their degeneracy. For $k_{b}<k_{b}^{\text {crit }}$, the $N_{\text {micro }}$ lowest energy semi-flexible polymer packings correspond to the $N_{\text {micro }}$ ground-state flexible polymer packings. In contrast, for $k_{b}>k_{b}^{\text {crit }}$ the energy landscape is sufficiently deformed that new low-energy packings (that do not belong to the set of $k_{b}=0$ ground-state polymer packings) are among the $N_{\text {micro }}$ lowest. For example, in Fig. 1 with $N_{\text {micro }}=8$, if the energy of polymer packing 9 drops below any of the energies of packings 1 through 8 , there is no one-to-one correspondence between the $N_{\text {micro }}$ lowest-energy microstates and $k_{b}>k_{b}^{\text {crit }}$.

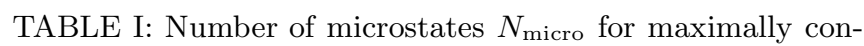
tacting flexible t-SHS packings, where $N_{\text {micro }}$ for $N>6$ includes a factor of two associated with packings possessing chiral enantiomers. [19] The number of contacts $N_{c}$ is the number of $\{i, j\}$ pairs with $r_{i j}<r_{c}$.

\begin{tabular}{lcc}
\hline \hline$N$ & $N_{c}$ & $N_{\text {micro }}$ \\
6 & 12 & 50 \\
7 & 15 & 700 \\
8 & 18 & 6429 \\
9 & 21 & 122060 \\
\hline \hline
\end{tabular}

All of the $N_{\text {micro }}$ distinguishable polymeric paths (microstates) through $\mathrm{N}$-monomer, $N_{c}$-contact flexible tSHS packings are obtained via the complete enumeration procedure described in Ref. [16]. Values of $N_{\text {micro }}$ for ground-state (maximal- $N_{c}$ ) flexible t-SHS packings of $6<N<9$ monomers are given in Table \.

Many computational models for polymers coarse-grain at the level of several monomers per coarse-grained spher- ical bead. However, since colloidal polymers are reasonably modeled by spheres with hard-core-like repulsions and short-range interactions, we coarse-grain at the level of one sticky sphere per colloidal monomer. Thus our range of $N$ is appropriate for modeling experiments like those in Refs. [4, 5], which studied colloidal polymers with $N \leq 8$.

The pair potential for t-SHS polymers with spherical monomers of diameter $D$ is [16, 20]:

$$
U_{s s}(r)=\left\{\begin{array}{cl}
\infty, & r<D \\
-\epsilon, & r=D \\
0, & r>D, \text { noncovalent } \\
\infty, & r>D, \quad \text { covalent }
\end{array}\right.
$$

where $r$ is the center-to-center separation between monomers and $-\epsilon$ is the contact energy. In Eq. 1. the term "covalent" ("noncovalent") refers to monomers that are (not) chemically adjacent and permanently connected. For numerical calculations, we employ a continuous version of $U_{s s}(r)$ with short-range attractive interactions and harmonic bond-length constraints illustrated in Fig. 2(a),

$$
U_{c}(r)=\left\{\begin{array}{cc}
-\epsilon+\frac{k_{c}}{2}\left(\frac{r}{D}-1\right)^{2} & , r<r_{c} \\
0 \quad, r \geq r_{c},
\end{array}\right.
$$

where $k_{c}$ is in units of $\epsilon, r_{c} / D=\infty$ for covalently bonded monomers and $r_{c}=r_{c}^{n c}=D\left(1+\sqrt{2 / k_{c}}\right)$ for noncovalently bonded monomers. Note that $U_{s s}(r)$ is the $k_{c} \rightarrow \infty$ limit of $U_{c}$ in Eq. 2

To model finite bending stiffness, we employ a harmonic bond-angle potential used in many previous computational studies of organic molecules, peptides, and proteins [21, 22]:

$$
U_{\mathrm{bend}}^{i}=\frac{k_{b}}{2} \frac{\left(\theta^{i}-\theta_{e q}^{i}\right)^{2}}{\pi^{2}}
$$

The bond angle $\theta^{i}$ between adjacent monomers $(i, i+1$, $i+2)$ is defined as $\theta^{i}=\pi-\cos ^{-1}\left[\left(\vec{b}_{i} \cdot \vec{b}_{i+1}\right) /\left|b_{i} b_{i+1}\right|\right]$, with $\vec{b}_{i}=\vec{r}_{i+1}-\vec{r}_{i} . U_{\text {bend }}^{i}$ is minimized at the equilibrium bond angle $\theta^{i}=\theta_{e q}^{i}$, and $k_{b}$ is expressed in units of $\epsilon$.

The total potential energy of a $N$-monomer chain is

$$
\begin{aligned}
U_{\text {chain }} & =U_{c}^{\text {tot }}+U_{b}^{\text {tot }} \\
& =\sum_{i=1}^{N-1} \sum_{j>i}^{N} U_{c}\left(r_{i j}\right)+\sum_{i=1}^{N-2} U_{\text {bend }}^{i}
\end{aligned}
$$

For $k_{b}=0$ and the limit $k_{c} \rightarrow \infty$, configurations corresponding to local minima of $U_{\text {chain }}$ are clearly identical to those obtained in studies of flexible t-SHS packings with no bond-angle interactions, i.e. all $N$-monomer, $N_{c^{-}}$ contact microstates have $U_{\text {chain }}=-N_{c} \epsilon$. 23]

Bond-angle interactions break this degeneracy; $U_{\text {chain }}$ increases by different amounts for each microstate. For t-SHS packings, to leading order (LO), the increase in 


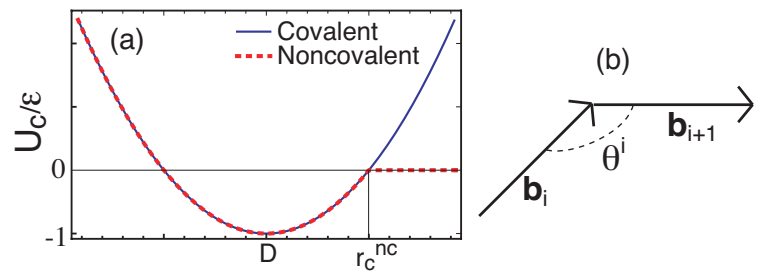

FIG. 2: Schematic of (a) the short-range attractive and harmonic bond-length potentials $U_{c}(r)$ (Eq. 2) and (b) the definition of the bond angle $\theta^{i}$ for the harmonic bond-angle potential $U_{\text {bend }}^{i}$ (Eq. 3).

energy $U_{\text {chain }}\left(k_{b}\right)-U_{\text {chain }}\left(k_{b}=0\right)$ scales linearly with $k_{b}$. To see why this is so, note that for $k_{c} \rightarrow \infty$, increasing the bending stiffness does not change the structure of the polymer packings. As $k_{c}$ becomes very large, the energetic penalty for changing the intermonomer distances $r_{i j}$ for all pairs $\{i, j\}$ becomes correspondingly large. In this limit, changing any of the bond angles alters the intermonomer distances and necessarily implies contact-breaking $\left(r_{i j}>D\right)$ or intermonomer overlap $\left(r_{i j}<D\right) .[16]$

Each "reference" $k_{b}=0$ microstate possesses a set of $N-2$ reference bond angles $\left\{\theta_{1}^{r e f}, \theta_{2}^{r e f}, \ldots, \theta_{N-2}^{r e f}\right\}$. The LO prediction for the energy of the $j$ th microstate is

$$
U_{\text {chain }, \mathrm{LO}}^{j}=-N_{c} \epsilon+\frac{k_{b}}{2 \pi^{2}} c_{j}\left(N, \theta_{e q}\right)
$$

where

$$
c_{j}\left(N, \theta_{e q}\right) \equiv \sum_{i=1}^{N-2}\left(\theta_{i}^{r e f, j}-\theta_{e q}\right)^{2}
$$

and we assume $\theta_{e q}^{i}=\theta_{e q}$ for all $i$. Since all $c_{j}$ are available from the flexible t-SHS packings, LO predictions for the energy of polymer packings for arbitrary $k_{b}$ and $\theta_{e q}$ can be made using only information from the flexible reference polymer packings. Next-to-leading order (NLO) corrections to $U_{\text {chain }}$ are negative and are expected to scale as $k_{b} / k_{c}$. Below, we will analytically and numerically calculate the total energy $U_{\text {chain }}$ for the reference packings versus $k_{b}$ and determine $k_{b}^{\text {crit }}$ as a function of $N$ and $\theta_{e q}$.

\section{RESULTS}

Predictions for the minimum, maximum, and average energies of polymer packings $\left(U_{\min }, U_{\max }, U_{\text {avg }}\right)$ from Eq. (4) for $N=8$ and two physically relevant $\theta_{e q}$ are shown in Fig. 3. Polymers with tetrahedral bond-angle order have $\theta_{e q} \simeq \theta_{\text {tet }}=\cos ^{-1}(-1 / 3)=109.47^{\circ}$, while $\theta_{e q}=\pi$ corresponds to polymers with linear bond-angle order. The bond-angle energies are higher for $\theta_{e q}=\pi$ since the compact structure of small- $N$ flexible t-SHS packings allows few "straight trimers" (angles with $\theta^{i}=\pi$ ). To vali- date the leading-order expressions for the energy of semi-

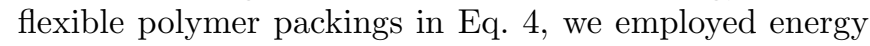
minimization techniques (described in the Appendix) to relax the t-SHS configurations in the presence of bondangle interactions. The $\mathrm{LO}$ expressions for the minimum $U_{\min }$, average $U_{\text {avg }}$, and maximum $U_{\max }$ chain energies closely agree with the numerical results over a wide range of $k_{b} / k_{c}$ as shown in Fig. 3. Both the numerical results and LO expressions for $U_{\text {chain }}$ are nearly linear in $k_{b} / k_{c}$ over the full range studied.

Note that for $\theta_{e q}=\theta_{\text {tet }},\left(U_{\max }+N_{c} \epsilon\right) /\left(U_{\min }+N_{c} \epsilon\right) \simeq 7$ because there are large bond angle fluctuations $\Delta \theta$ in the flexible t-SHS packings [16, 19, 24] as shown in Fig. [4. The variation in $\langle\theta\rangle$ and $\Delta \theta$ increases rapidly with $N$ (Fig. (4) and suggests that $k_{b}^{c r i t} \ll 1$.

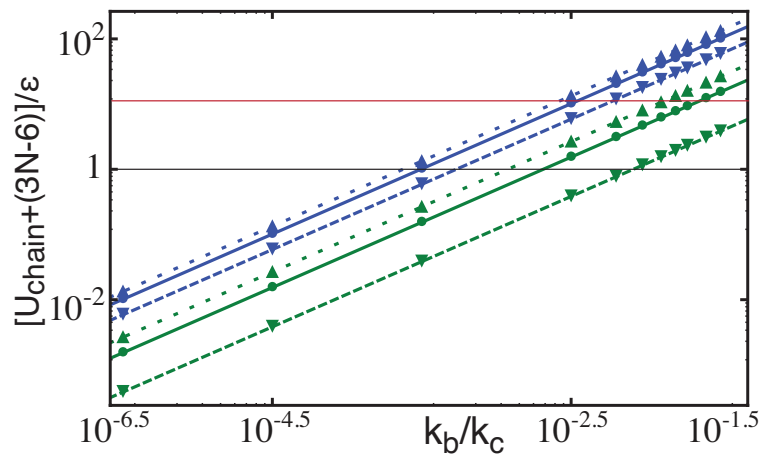

FIG. 3: Comparison of the leading-order expressions for the energy $U_{\text {chain }}$ of $N=8$ semiflexible polymer packings in Eq. 4 (dashed, $U_{\min }$; solid, $U_{\text {avg }}$; dotted, $U_{\max }$ ) to the numerical results for the energy of minimized t-SHS packings in the presence of bond-angle interactions (downward triangles, $U_{\min }$; circles, $U_{\text {avg }}$; upward triangles, $\left.U_{\max }\right)$ as a function of $k_{b} / k_{c}$. Results for $\theta_{e q}=\theta_{\text {tet }}$ and $\pi$ are shown in green and blue, respectively. $U_{\text {chain }} / \epsilon=-\left(N_{c}-1\right)$ and $U_{\text {chain }}=0$ are respectively indicated by the black and red horizontal lines.

To visually estimate $k_{b}^{\text {crit }}$ from Fig. [3] we note that freely-rotating chain conformations with no pair contacts and $\theta^{i}=\theta_{e q}$ for all bond angles have $U_{\text {chain }}=-(N-1) \epsilon$. Thus it is clear that $k_{b} / k_{c}>k_{b}^{\text {crit }} / k_{c}$ for $U_{\text {chain }}>0$, and that the critical bending stiffness $k_{b}^{\text {crit }}$ is below $k_{b}^{\text {crit }} / k_{c} \sim$ $10^{-1.5}$. A more refined (if heuristic) estimate of $k_{b}^{\text {crit }}$ can be obtained as follows. The $N_{\text {micro }}(N)$ flexible tSHS packings all possess $N_{c}(N)$ pair contacts (Table I) and energy $-N_{c}(N) \epsilon$. Any polymer packing with fewer than $N_{c}(N)$ pair contacts must have $U_{\text {chain }} \geq-\left(N_{c}-1\right) \epsilon$ since the bending energy is strictly positive. Conversely, any t-SHS polymer packing with $N_{c}$ pair contacts must correspond (in the sense of Fig. (1) to one of the $N_{\text {micro }}(N)$ flexible t-SHS packings. Therefore $k_{b}^{\text {crit }}\left(N, \theta_{e q}\right)$ is set by the largest $c_{j}\left(N, \theta_{e q}\right)$ (Eq. 5) for these packings and the condition that the reduced bending energy is less than unity, which gives

$$
k_{b}^{\text {crit }}\left(N, \theta_{e q}\right) \leq 2 \pi^{2} / c_{j}^{\max }\left(N, \theta_{e q}\right) .
$$

As shown in Fig. 5 , $k_{b}^{\text {crit }}$ decreases monotonically with 


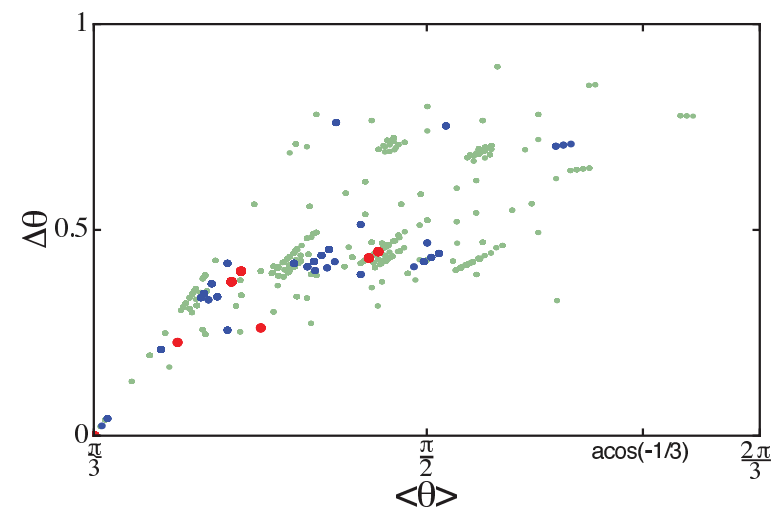

FIG. 4: Bond-angle dispersion for flexible t-SHS polymer packings. Each data point represents the mean bond angle $\langle\theta\rangle$ and the root-mean-square deviation $\Delta \theta$ for a single flexible t-SHS polymer packing. Large red, medium blue, and small green circles represent data for $N=6$. 7 , and 8 , respectively.

increasing $N$ for all $\theta_{e q} \lesssim \pi / 2$ and $\theta_{e q} \gtrsim 2 \pi / 3$, which stems from the increasing angular dispersion with $N$ shown in Fig. 4. In addition, for increasing $\theta_{e q}, k_{b}^{\text {crit }}$ reaches a peak at $\theta_{\max }$ that increases with $N$, and then decreases for $\theta_{e q}>\theta_{\max }$. For the $N$ considered here, the peak occurs at a rather small $\theta_{\max } \lesssim \pi / 2$ because of the compactness of the flexible t-SHS reference polymer packings. We expect the decrease in $k_{b}^{\text {crit }}$ with increasing $N$ to continue for $N>9$.

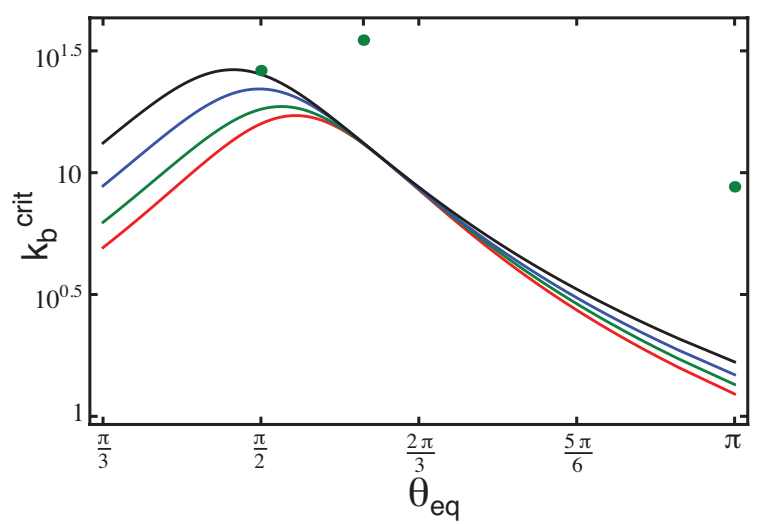

FIG. 5: Estimates of $k_{b}^{\text {crit }}\left(N, \theta_{e q}\right)$ from Eq. 6 for $N=6$ (black line), 7 (blue line), 8 (green line), and 9 (red line) and from MD simulations of finite- $k_{b}$ t-SHS polymers with $N=8$ (green circles).

To check the analytic prediction of Eq. 6 for $k_{b}^{\text {crit }}$, we performed MD simulations of semiflexible t-SHS chain collapse for $N=8$. Monomers interact via the pair and bond-angle potentials in Eqs. 22and 3, with $k_{c}=3200$ and a range of $\theta_{e q}$ and $k_{b}$. For each $\theta_{e q}, k_{b}$, and $k_{c}, N_{\mathrm{S}}=1000$ systems with independent self-avoiding random walk initial polymer configurations were slowly quenched from high temperature $\left(k_{B} T_{i}=4 \epsilon\right)$ to $k_{B} T_{f}<10^{-10} \epsilon$ using an isokinetic thermostat [25] and quench protocol
$T=T_{i} \exp \left[-t /\left(10^{3} \tau\right)\right]$, where $\tau=\sqrt{m D^{2} / \epsilon}$ is the unit of time and $m$ is the monomer mass. 26] An overestimate of $k_{b}^{\text {crit }}$ can be obtained by measuring the lowest $k_{b}$ at which the minimum energy collapsed states obtained via MD have chain energies below $U_{\min }$; these cannot correspond to any of the $N_{\text {micro }}$ flexible t-SHS inherent structures. Note that in the sticky-hard-sphere limit, $k_{b}^{\text {crit }}$ is set by the structure of t-SHS packings (i.e. the number $N_{c}(N)$ and types of inter-monomer contacts).

A comparison of $k_{b}^{\text {crit }}$ from Eq. [6 and estimates from the MD simulations is shown in Fig. 5 for several $\theta_{e q}$. The green circles illustrate the lowest bending stiffnesses $k_{b}^{*}$ at which polymer packings with energies lower than $U_{\min }$ are formed. In all cases, the polymer packings from MD simulations with energies $U_{\text {chain }}<U_{\text {min }}$ possess 23 fewer pair contacts than the flexible t-SHS inherent structures and have $k_{b}^{*}$ such that $\left[U_{\max }\left(k_{b}^{*}\right)+(3 N-6)\right] \simeq$ $(2-3) \epsilon$. Thus, energetically favorable rearrangements of t-SHS polymer packings due to finite bending stiffness typically require breaking two or three pair contacts.

We now return to the picture of Fig. 1 and analyze how the introduction of nonzero bending stiffness alters the low-lying energy landscapes of t-SHS polymers. Figure 6(a) compares the variation of the structural and angular energy degeneracy splittings for $4 \leq N \leq 9$ and $\theta_{e q}=\pi$ in the limit $k_{b} \rightarrow 0$. The reference t-SHS packings can be classified into $\mathcal{M}(N)$ distinguishable (structurally nonisomorphic 16]) "macrostates" possessing different shapes, i.e. sets of squared interparticle distances $\left.\left\{r_{i j}^{2}\right\}\right)$ [16], and $\mathcal{M}(N)$ grows exponentially with $N$. In general, the different polymeric paths through any given macrostate have many different combinations of angles (Fig. 4), and thus, at finite $k_{b}$, different energies. The number $\mathcal{S}(N)$ of distinct angular energy levels $U_{b}^{\text {tot }}$ for any given $\theta_{e q}$ also increases exponentially with $N$ and scales roughly as $N_{\text {micro }} / \mathcal{M}$. Figure 6(b) illustrates the range of energy levels, $R=U_{\max } / U_{\min }$, for $N=8$ t-SHS polymers in the $k_{b} \rightarrow 0$ limit. In contrast to the exponential increase in the number of distinct energy levels, $R$ increases only (roughly) linearly with $N$. This can be understood as follows. Since $U_{\max }$ has an upper bound

$$
U_{\max }^{t h}=(N-2)\left(k_{b} / 2 \pi\right)(2 \pi / 3)^{3}
$$

imposed by steric constraints, the spacing between energy levels decreases as $\mathcal{S}$ increases. This is illustrated in Figure 6(c), which presents results for the distribution $P\left(U_{b}^{\text {tot }} / U_{\max }^{t h}\right)$ of the total angular energies relative to $U_{\max }^{\text {th }}$ for $6<N<8$. Results for all $N$ show $\delta$-functionlike peaks, and the spacing between energy levels decreases as $N$ increases. The distribution $P\left(U_{b}^{\text {tot }} / U_{\max }^{t h}\right)$ also broadens as $N$ increases. We have also examined $P\left(U_{b}^{t o t} / U_{m a x}^{t h}\right)$ for $\theta_{e q}=\theta_{t e t}$. While the distribution shifts to lower values of $U_{b}^{\text {tot }}$ since $\theta_{\text {tet }}$ is a commonly occurring angle in t-SHS packings, qualitative trends with $N$ are similar. Note that Fig. 6 shows analytic results calculated from the values of $c_{j}\left(N, \theta_{e q}\right)$. At finite $k_{b}<k_{b}^{\text {crit }}$, numerical results for $P\left(U_{b}^{\text {tot }} / U_{\max }^{t h}\right)$ show that packings with the same $\left\{\theta^{i}\right\}$ but topologically distinct orderings of 

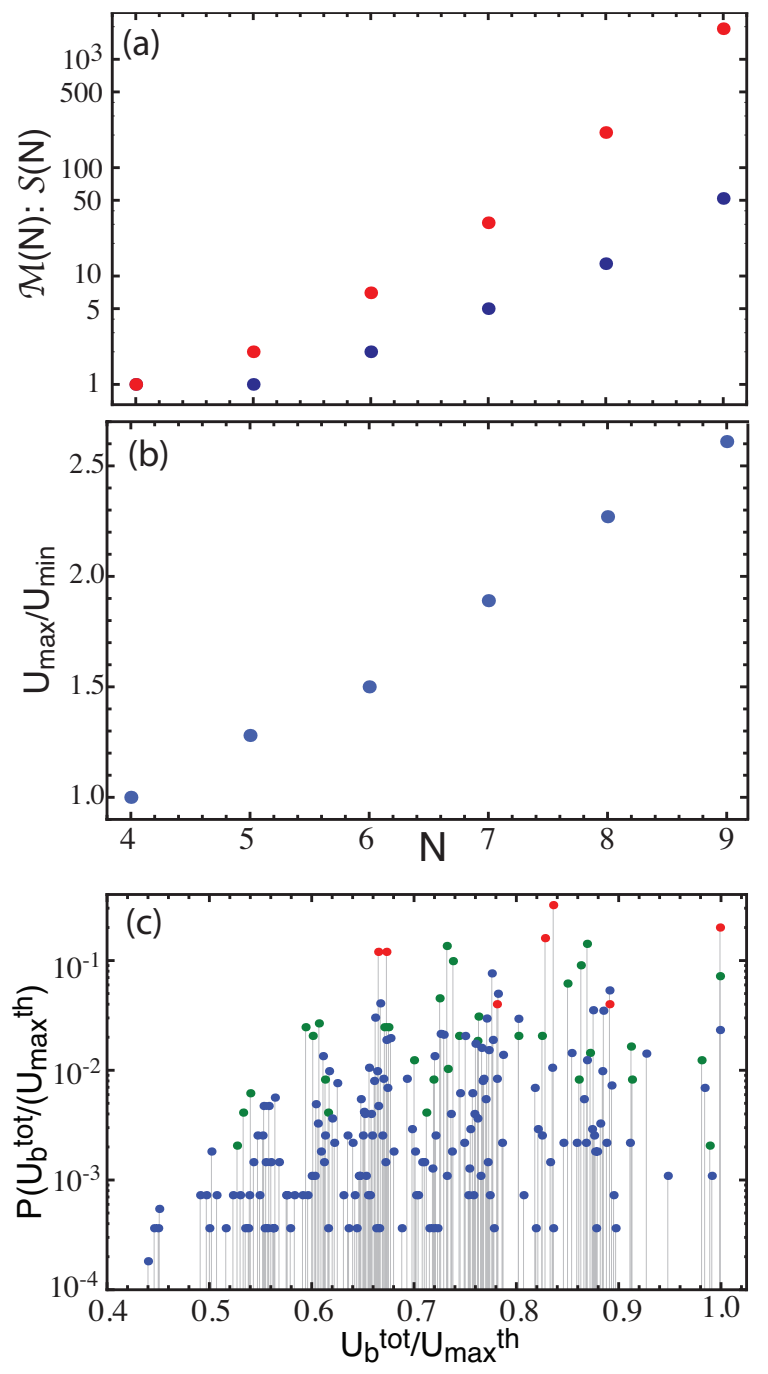

FIG. 6: (a) Number of nonisomorphic packings (macrostates) $\mathcal{M}(N)$ [16] (blue circles) and non-degenerate angular energy levels $\mathcal{S}(N)$ (red circles), and (b) ratio $U_{\max } / U_{\min }$ plotted versus $N$ for $\theta_{e q}=\pi$, in the limit $k_{b} \rightarrow 0$. (c) The distribution of angular energies $P\left(U_{b}^{\text {tot }} / U_{\max }^{\text {th }}\right)$ for $N=6$ (red) 7 (green), and 8 (blue).

the $\theta^{i}$ relax differently towards $\theta_{e q}$, and the distribution of angular energies becomes more continuous.

Next we examine features of the low-lying energy landscape for packings with $k_{b} \simeq k_{b}^{c r i t}$. Figure 7 (a) contrasts the probability distributions $P\left(U_{b}^{\text {tot }} / U_{\max }^{\text {th }}\right)$ for systems with $N=8$ and $\theta_{e q}=\theta_{\text {tet }}$ in the limit $k_{b} \rightarrow 0$, for two finite values of $k_{b}: k_{b}=1 \ll k_{b}^{c r i t}$, and $k_{b}=10^{1.5} \simeq k_{b}^{*}$. Results for the larger two $k_{b}$ are obtained using the same MD protocol described above, while results for $k_{b} \rightarrow 0$ are evaluated analytically using $c_{j}\left(N, \theta_{e q}\right)$. Results for $k_{b} \rightarrow 0$ and $k_{b}=1$ are essentially equivalent in that all packings correspond to t-SHS packings. Small differences arise from the MD quench protocol, which favors formation of packings with lower $U_{b}^{\text {tot }}$.

Our results from the MD simulations for $k_{b} \simeq k_{b}^{*}$ il-
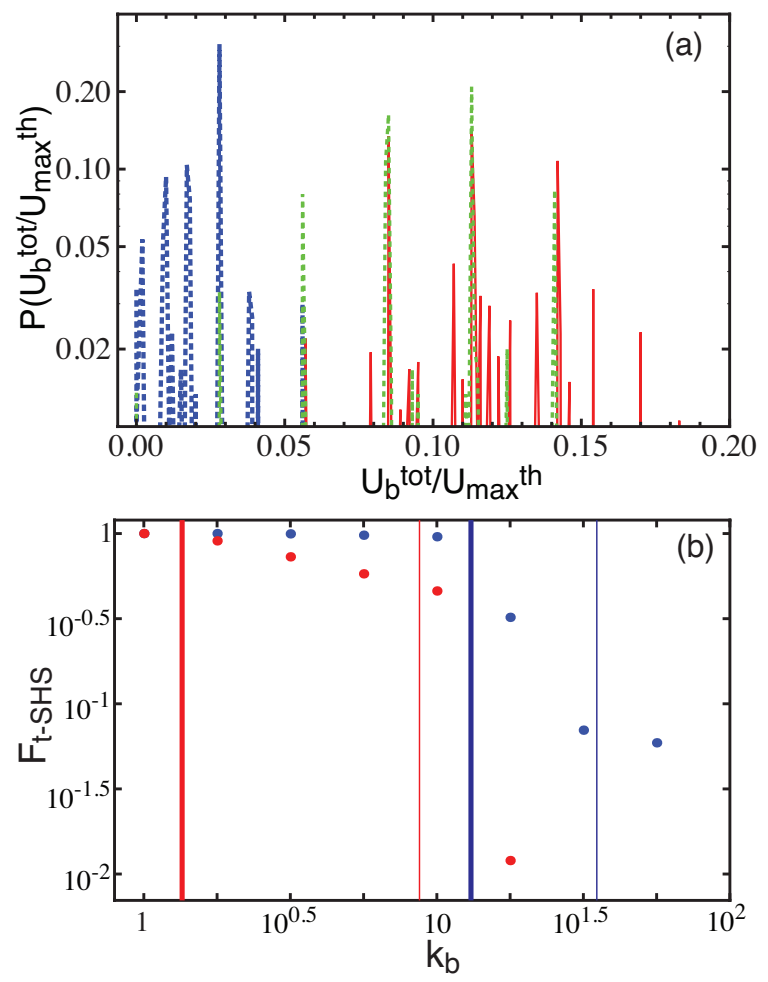

FIG. 7: (a) Distributions $P\left(U_{b}^{\text {tot }} / U_{\max }^{\text {th }}\right)$ of the total angular energy $U_{b}^{\text {tot }}$ relative to $U_{\max }^{\text {th }}$ in the $k_{b} \rightarrow 0$ limit (red), $k_{b}=1$ (green) and $10^{1.5}$ (blue) for $N=8$ and $\theta_{e q}=\theta_{\text {tet }}$. (b) Fraction $F_{t-S H S}$ of collapsed packings corresponding to t-SHS microstates as a function of $k_{b}$ for $\theta_{e q}=\pi$ (red circles) and $\theta_{\text {tet }}$ (blue circles). The heavy and light solid lines correspond to estimates of $k_{b}^{\text {crit }}$ from Eq. 6] and MD estimates of $k_{b}^{*}$ (Fig. [5), respectively.

lustrate that significant changes occur in the low-lying energy landscape for semi-flexible t-SHS polymers. The distribution $P\left(U_{b}^{\text {tot }} / U_{\text {max }}^{\text {th }}\right)$ overlaps those for $k_{b} \rightarrow 0$ only at the lowest peak $\left(U_{b}^{\text {tot }} / U_{\max }^{t h} \simeq\right.$.058). The remainder of the collapsed states have lower $U_{b}^{\text {tot }} / U_{\max }^{\text {th }}$ and do not correspond to flexible t-SHS packings. These states possess fewer pair contacts $\left(N_{c}<18\right)$ and hence greater freedom to reduce $U_{b}^{\text {tot }}$ by relaxing bond angles toward $\theta=\theta_{\text {eq }}$. Figure $7(\mathrm{~b})$ shows the fraction of collapsed MD-quenched configurations that form ground-state flexible t-SHS polymer packings as a function of $k_{b}$. We find that the low-lying energy landscape for t-SHS polymers changes dramatically in the $k_{b}^{c r i t}<k_{b} \lesssim k_{b}^{*}$ regime when the structural properties of the lowest $N_{\text {micro }}$ energy minima become significantly different from flexible t-SHS packings.

\section{CONCLUSIONS}

In this manuscript, we characterized tangent-stickyhard-sphere polymer packings with finite bending stiffness. We have shown that t-SHS polymers possess the 
same low-lying energy landscapes for $k_{b}<k_{b}^{\text {crit }}$, where $k_{b}^{c r i t}$ depends strongly on the equilibrium bond angle $\theta_{e q}$ and decreases with increasing degree of polymerization $N$. Angular interactions introduce new energy levels (compared to flexible t-SHS packings), whose number increases exponentially with $N$. As $k_{b}$ increases above $k_{b}^{c r i t}$, the t-SHS energy landscape breaks down as a useful reference for the low-lying energy-landscape of compact finite-stiffness polymers, revealing an interesting regime in which minimizing pair and bond-angle energies compete. In future studies, we will enumerate and characterize the structural and mechanical properties of minimal energy semiflexible polymer packings with $k_{b}>k_{b}^{\text {crit }}$ using advanced sampling techniques.

Many recent theoretical studies have examined the structure of collapsed, flexible polymer chains (e.g. Refs. [6-15]). It would be interesting to check how these are affected by a small but finite bending stiffness. For example, even a small $k_{b}$, since it would alter the local ground-state structure of the polymer packings, seems likely to produce a strong effect on the jamming transition of densely packed collapsed polymers, which for fully flexible chains occurs [7 9] at random close packing [27] as for monomers.

Support from NSF Award No. DMR-1006537 is gratefully acknowledged. This work also benefited from the facilities and staff of the Yale University Faculty of Arts and Sciences High Performance Computing Center and NSF grant No. CNS-0821132 that partially funded acquisition of the computational facilities.

\section{Appendix A: Structure solver for packings with finite $k_{c}$ and $k_{b}$}

We find the mechanically stable, minimal energy configuration $\left\{\vec{r}_{\text {min }}\right\}$ closest to each flexible packing $\left\{\vec{r}_{f l e x}\right\}$ by starting from configurations $\left\{\vec{r}_{\text {pert }}\right\}$ representing small random perturbations 28] away from $\left\{\vec{r}_{\text {flex }}\right\}$, and minimizing $U_{\text {chain }}$. Energy minimization is carried out using the "dog-leg" method [29], which is a combination of gradient descent and Newton's method [30]. Our numerical algorithm is schematically depicted in Figure 8 .

For finite $k_{b}$, the first spatial derivatives of $U_{\text {chain }}$ evaluated at $\{\vec{r}\} \neq\left\{\vec{r}_{\text {min }}\right\}$ are generally nonzero. We approach $\{\vec{r}\}=\left\{\vec{r}_{\min }\right\}$ using gradient descent and a second-order expansion of $f(\{\vec{r}\}) \equiv U_{\text {chain }}(\{\vec{r}\})$. This descent iteratively reduces $f$ using

$$
f_{n}^{*}=f_{n}-\alpha \nabla f_{n},
$$

where $f_{n}$ is the value of $f$ at the nth minimization step, $f_{n}^{*}$ is $U_{\text {chain }}$ at the "knee" of the dog-leg [31], the optimal step length is 32]

$$
\alpha=\frac{\nabla f(\nabla f)^{T}}{\nabla f H(f)(\nabla f)^{T}},
$$

and $H\left(f_{n}\right)$ is the $3 N \times 3 N$ Hessian matrix of $f_{n}$. After the gradient descent step, we perform a least-squares Newton iteration on $\nabla f_{n}$ using step-length control [30] (Box 1):

$$
f_{n+1}=f_{n}^{*}-\left(H^{T}\left(f_{n}^{*}\right) H\left(f_{n}^{*}\right)\right)^{-1} H^{T}\left(f_{n}^{*}\right) \nabla f_{n}^{*} .
$$

If the Frobenius norm $\left\|\nabla f_{n+1}\right\|_{2}$ of $\nabla f_{n+1}$ decreases (i.e. $\left.\left\|\nabla f_{n+1}\right\|_{2}<\left\|\nabla f_{n}^{*}\right\|_{2}\right)$, we check whether $f_{n+1}<f_{n}^{*}$. If $f_{n+1}>f_{n}^{*}$, we set $f_{n+1}=f_{n}^{*}$; if $f_{n}^{*} \geq f_{n}$, the gradient descent has failed and we set $f_{n}^{*}=f_{n}$ in Eq. A3. We then check whether $\left\|\nabla f_{n+1}\right\|_{2} \leq\left\|\nabla f_{n}^{*}\right\|_{2}$; if so, Equations A1 A3 represent one complete minimization step taking $f_{n} \rightarrow f_{n+1}$ (Boxes 1-2). Otherwise, if $\left\|\nabla f_{n+1}\right\|_{2}>\left\|\nabla f_{n}^{*}\right\|_{2}$ the minimization algorithm has failed (Box 3); however, this occurs only for $k_{b}$ much larger than those considered here.

The minimization procedure reduces the first spatial derivatives of $U_{\text {chain }}$ to zero. When this condition is satisfied $(\nabla f=0)$, we check whether $\{\vec{r}\}$ represents a mechanically stable solution, i.e. whether all $3 N-6$ nontrivial eigenvalues of $H$ are positive (Box 4). Saddle-point solutions are avoided (when encountered) by perturbing coordinates along a negative eigenvector of the Hessian matrix (Box 5).

Should the solver reach the maximum number of iterations for a particular $\left\{\vec{r}_{\text {pert }}\right\}$ (MAXRUNS; Box 6) we attempt to minimize a different $\left\{\vec{r}_{\text {pert }}\right\}$, for up to MAXITS different $\left\{\vec{r}_{\text {pert }}\right\}$ (Box 7). We found that MAXITS = 1000 different $\left\{\vec{r}_{\text {pert }}\right\}$ with a maximum of 50 iterations per $\left\{\vec{r}_{p e r t}\right\}$ to be sufficient to minimize the energy or determine that no minimum that preserves the adjacency matrix exists for the $N \leq 9$ polymer packings considered here.

Finally, note that the time required for our structure solver to find energy minima is insignificant for a given $k_{b}$ and $\theta_{e q}$. However, two factors limit the present study to $N=9$ : (a) The time required to enumerate the flexible perturbative basis increases faster than exponentially with $N$, as reported in Refs. [16, 24]. (b) While flexible polymer packings have been generated for $N$ up to 11 , the ground state packings for $N=10$ and 11 show much less structural diversity (e.g. fewer possible $\theta^{i}$ ) and hence are less suitable for perturbative studies of the effect of angular stiffness.
[1] P. J. Flory, C. A. J. Hoeve, and A. Ciferri, J. Poly. Sci. 34, 337 (1959).

[2] G. N. Ramachandran and V. Sasisekharan, Adv. Prot.
Chem. 23, 284 (1968).

[3] F. M. Richards, Ann. Rev. Biophys. Bioeng. 6, 151 (1977). 


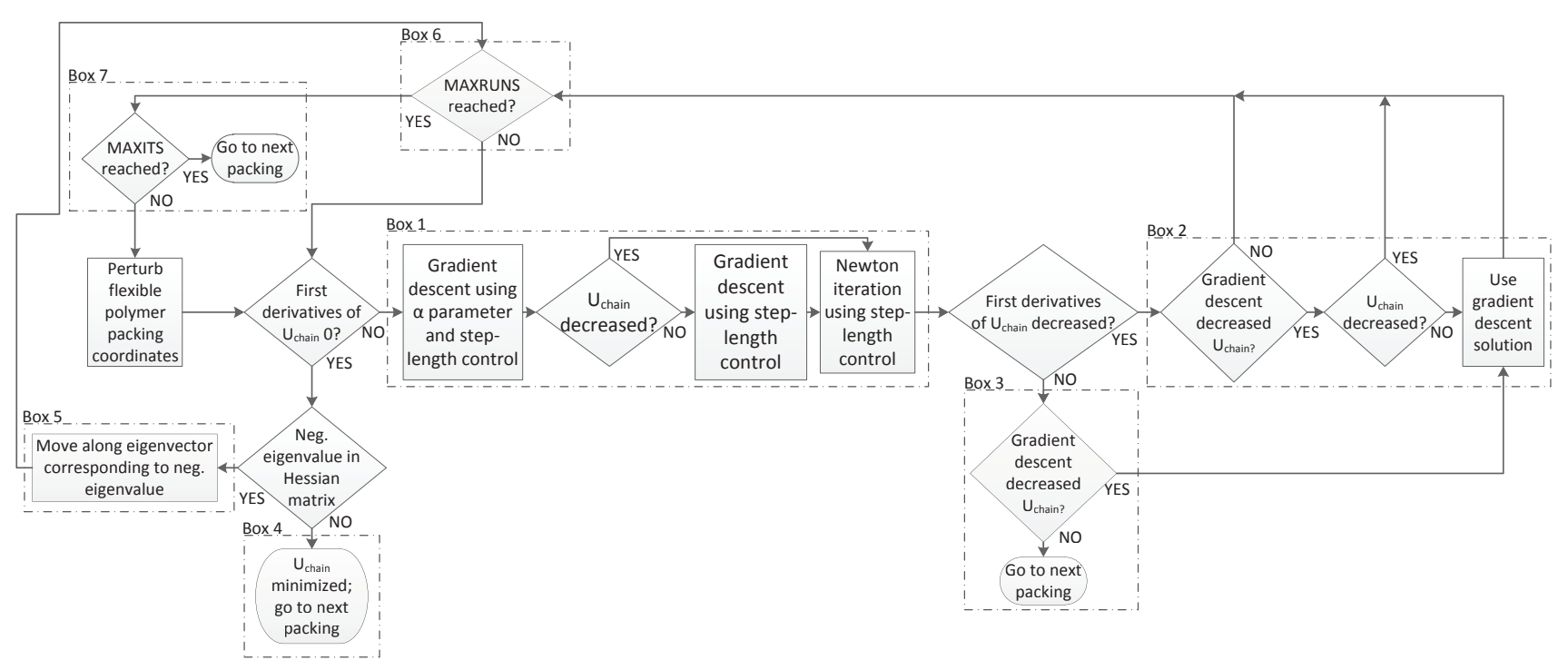

FIG. 8: Flowchart depicting the algorithm used to find the mechanically-stable, minimum-energy configuration $\left\{\vec{r}_{m i n}\right\}$ closest to each flexible packing configuration $\left\{\vec{r}_{f l e x}\right\}$.

[4] S. Sacanna, W. T. M. Irvine, P. M. Chaikin, and D. J. Pine, Nature 464, 575 (2010).

[5] M. J. Solomon, Nature 464, 496 (2010).

[6] M. P. Taylor, J. Chem. Phys. 118, 883 (2003).

[7] K. Foteinopoulou, N. C. Karayiannis, M. Laso, M. Kröger, and M. L. Mansfield, Phys. Rev. Lett. 101, 265702 (2008).

[8] N. C. Karayiannis, K. Foteinopoulou, and M. Laso, J. Chem. Phys. 130, 164908 (2009).

[9] M. Laso, N. C. Karayiannis, K. Foteinopoulou, M. Laso, M. L. Mansfield, and M. Kröger, Soft Matter 5, 1762 (2009).

[10] M. P. Taylor, W. Paul, and K. Binder, J. Chem. Phys. 131, 114907 (2009).

[11] M. P. Taylor, W. Paul, and K. Binder, Phys. Rev. E 79, 050801 (2009).

[12] D. T. Seaton, T. Wüst, and D. P. Landau, Phys. Rev. E 81, 011802 (2010).

[13] G. T. Evans, J. Chem. Phys. 134, 044902 (2011).

[14] R. S. Hoy and C. S. O'Hern, Soft Matter 8, 1215 (2012).

[15] S. Růžička, D. Quigley, and M. P. Allen, Phys. Chem. Chem. Phys. 14, 6044 (2012).

[16] R. S. Hoy and C. S. O'Hern, Phys. Rev. Lett. 105, 068001 (2010).

[17] S. Schnabel, M. Bachmann, and W. Janke, J. Chem. Phys. 131, 124904 (2009).

[18] F. H. Stillinger, Science 267, 1935 (1995).

[19] N. Arkus, V. N. Manoharan, and M. P. Brenner, Phys. Rev. Lett. 103, 118303 (2009).

[20] S. B. Yuste and A. Santos, Phys. Rev. E 48, 4599 (1993).

[21] P. C. Cross and J. H. Van Vleck, J. Chem. Phys. 1, 350 (1933).

[22] S. Toxvaerd, J. Chem. Phys. 107, 5197 (1997).

[23] We employ a large value of $k_{c}$ (3200), which because of its small $r_{c}$, is in the SHS limit and allows a one-to-one map- ping between flexible t-SHS polymer packings and degenerate ground states in the $k_{b} \rightarrow 0$ limit. For softer interactions with larger values of $r_{c}$, second-nearest-neighbor pair interactions would break this degeneracy. However, an analysis similar to that presented below but taking account of this degeneracy-breaking could be performed.

[24] R. S. Hoy, J. Harwayne-Gidansky, and C. S. O'Hern, Phys. Rev. E 85, 051403 (2012).

[25] D. Brown and J. H. R. Clarke, Mol. Phys. 51, 1243 (1984).

[26] The quench rate employed is close to the slow-quench limit for the $k_{c}=3200$.

[27] S. Torquato, T. M. Truskett, and P. G. Debenedetti, Phys. Rev. Lett. 84, 2064 (2000).

[28] Each monomer position is perturbed by a maximum amplitude $|\delta \vec{r}|_{\max }=0.2$, which was chosen to optimize the efficiency of the structure solver while satisfying the requirement $|\delta \vec{r}|_{\max }<(\sqrt{2}-1) / 2$ for preserving chain topology.

[29] J. E. Dennis, Jr. and R. B. Schnabel, Numerical Methods for Unconstrained Optimization and Nonlinear Equations (Classics in Applied Mathematics, v. 16: Soc. for Industrial \& Applied Math., 1996).

[30] W. Press, S. A. Teukolsky, W. T. Vetterling, and B. P. Flannery, Numerical Recipes: The Art of Scientific Computing (3rd ed.) (Cambridge University Press, 2007).

[31] In the event that $U_{\text {chain }}$ does not decrease, we attempt gradient descent with step-length control without the $\alpha$ parameter, i.e. $f_{n}^{*}=f_{n}-\nabla f_{n}$.

[32] M. Powell. A Hybrid Method for Nonlinear Equations. In P. Rabinowitz, editor, Numerical Methods for Nonlinear Algebraic Equations, pages 87144. Gordon and Breach Science, London, 1970. 